\title{
A Cross Sectional Study to Assess Perception and Behavior towards COVID-19 Vaccine Among Students and Faculties of Nursing Colleges at Anand and Kheda Districts, Gujarat.
}

\author{
Kailash Nagar ${ }^{1}$, Christian Selina ${ }^{2}$, ParmarAnushka ${ }^{3}$, Patel Devanshi ${ }^{4}$, Bhatt Dhruti ${ }^{5}$, Dalwadi \\ Jinal $^{6}$, Brahmbhatt Manjari ${ }^{7}$, Hiral Janki ${ }^{8}$. \\ ${ }^{1}$ Assistant Professor \& HOD of Community Health Nursing Department, Dinsha Patel College of \\ Nursing, Nadiad. \\ 2-8 \\ Final year B.Sc. Nursing Students, Dinsha Patel College of Nursing, Nadiad.
}

Corresponding Author: - Mr. Kailash Nagar (Email Id: dpcnkailash@ gmail.com)

https://orcid.org/0000-0002-0564-2761, ResearcherID: AAM-6294-2021

Address:- Dinsha Patel College of Nursing, Behind Hyundai Showroom, College road, Nadiad, Kheda District, Gujarat- 387001.

\section{ABSTRACT}

Introduction: On 11 March 2020, World Health Organization (WHO) announce that COVID-19 as a pandemic health problem [1,2]. According to report on 25 January 2021, worldwide cases reached to 100 million [3]. The first confirmed case of COVID-19 in India was reported on 30, January 2020 in Kerala and affected individual had come from Wuhan, china [4]. Corona virus is spread over 220 countries across the world [2]. In order to prevent corona virus all the countries are taken initiative to prepare effective vaccine against corona virus. There is not any effective treatment is available for corona so far vaccine is the key preventive aspect against corona virus. There are presently above 125 vaccines go through preclinical investigation for covid-19[5].

India has develop two types of vaccine (Covaxin and covidshield) in primary phase and from 01 January 2021, Indian government started vaccination namely Covidshield to health workers (front line workers) [6]. So in primary stage target set to cover 30 million health workers followed by policeman and old age peoples to be vaccinated against corona [6]. During primary phase of corona vaccine we don't have appropriate research and literature, about side effects and how far vaccine is reliable that why due so some miner side effect and negative media publicity peoples are very scared to take vaccine. So few peoples were started denial get vaccinated.

Aims The current study is aimed to assess the perception and behavior towards Covid-19 vaccine among students and faculties of nursing colleges those who have taken vaccine against corona. The researcher wan to explore the positivity through the research result to reduce the negative mindset of the peoples toward corona vaccine, Because in India few peoples has fear to take vaccine against corona due to negative media publicity and scared of side effect.

Objective 1. To assess the existing level of perception toward COVID 19 vaccine among students and faculties of Nursing colleges at Anand and Kheda Districts. 
medRxiv preprint doi: https://doi.org/10.1101/2021.07.18.21260710; this version posted July 29, 2021. The copyright holder for this preprint

2. To assess the behavior towards COVID 19 vaccine among students and faculties of Nursing colleges at Anand and Kheda Districts.

3. To find out the association between selected socio-demographic variables and perceptions towards COVID 19 vaccine.

\section{Methodology}

Design and Setting Descriptive cross sectional survey research design was used and non-probability (snowball) sampling method was used to drawn samples through online Google form [7]. Due to Covid19 pandemic situation research has adopted online snowball sampling method, where after tool validation from various subject experts, all questions were plots on Google form and inform consent form also has been conducted online prior to data collection from the samples[8].

Prior to data collection written setting permission obtain from nursing colleges principals, for the data collection researcher were selected total 03 nursing institutes which were namely Dinsha Patel College of Nursing, Vinayaka College of Nursing, Nadiad, Vinayaka College of Nursing, Anand, and Zydus College of nursing, Anand. The total sample size was 254 nursing college students and faculties.

The tool consist of following Section-01 Demographic variables, section-02 Nursing students and faculties information related to covid-19 vaccination during $1^{\text {st }}$ and $2^{\text {nd }}$ dose and Section-3 Questions related to perception and behaviour towards COVID 19 vaccine.

Statistical Analysis used Descriptive statistics applied where, data were analyzed by using SPSS software, and Frequency, percentage, tables etc. were used to represent the statistical data in the tables and graph and figure. Chi-square test was used to assess the significant as sociation between the demographic and level of perception to test the hypothesis.

Results Out of 254, 245(96.45\%) were belong age 17-25 years, 219(86.22\%) were females, 53(20.87\%) were study Diploma course and 178(70.08\%) were study degree course, 223(87.79\%) belong to Hindu, religion, 227(89.37\%) were Unmarried, 134(52.75\%) were from urban area, and rest $120(47.24 \%)$ belongs to Rural area. Sources of information about COVID 19 vaccine 109(42.92\%) got from online media, 44(17.32\%) from television, 243(95.67\%) received free of cost corona vaccine, 199(78.35\%) mindset was not influenced by negative media publicity about vaccine, 248(97.63\%) do not have any comorbidities. 219(86.22\%) taken Covid-19 vaccine empty stomach.

$221(87 \%)$ of samples were taken willingly vaccine, 205(80.71\%) of samples were received covidshield vaccine and others 49(19.29\%) were taken Covaxin, 109(42.91\%) samples noticed mild fever, 53(20.87\%) samples noticed moderate fever, 18(7.08\%) noticed severe fever and rest 74(29.13\%) didn't noticed fever.

Conclusions Regarding perception and behaviour towards COVID 19 vaccine, the majority of samples has good perception and behaviour, $74.00 \%$ has good perception and only 23.00 had moderate to poor perception, majority of participant were willingly taken vaccine and agree to recommend to others, not evidence any serious side effect due to vaccination.

KEYWORDS Covaxin, Covidshield, DPCN, WHO, Nursing, College, Fever. 
medRxiv preprint doi: https://doi.org/10.1101/2021.07.18.21260710; this version posted July 29, 2021. The copyright holder for this preprint

\section{INTRODUCTION:}

On 30 January, World Health Organization (WHO) 2020, announce COVID-19 as a public health crisis and afterwards On 11 March 2020, World Health Organization (WHO) announce the corona virus disease 2019 a pandemic (COVID19) [1].

Vaccination was one of the greatest costefficient, inhibitory actions [9]. Vaccines were the upmost essential public wellness actions and highly successful method to save public from covid-19 [10]. The world is presently working for the quick evolution of the COVID 19 vaccine. A successful COVID 19 vaccine should be useful, effective, set free from any side effect and affordable for local people in the world $[11,14]$.

There are presently above 125 vaccines go through pre-clinical investigation for covid-19. The vaccines are than go into three phases of clinical tests, India has already rolled out a huge coronavirus effort to utilize two vaccines, Covishield and Covaxin $[5,16]$.

The covid-19 vaccine was introduced on $16^{\text {th }}$ January, 2021. Health personnel and frontline workers were the first group who get the opportunity to get COVID-19 vaccine and after them individuals who are above 50 years of age and individuals who are under 50 years and suffering from co-morbidity conditions were the second group for COVID-19 vaccination [6].

There were two doses of covid-19 vaccine which would be offered in 28 days' gap. The efficiency of vaccine starts later on 14 days of taking the second dose. The covid-19 vaccine was extremely fruitful against covid-19[11]. some experts declare that the vaccine protected against covid-19 in $62 \%$ of those who received two full doses and $90 \%$ of those who initially received half dose $[12,13,15]$.

OBJECTIVE: 1 . To assess the existing level of perception toward COVID 19 vaccine among students and faculties of Nursing colleges at Anand and Kheda Districts.

2. To assess the behavior towards COVID 19 vaccine among students and faculties of Nursing colleges at Anand and Kheda Districts.

3. To find out the association between selected socio-demographic variables and perceptions towards COVID 19 vaccine.

\section{MATERIALS AND METHODS}

Research Approach: - Non Experimental,

Descriptive survey approach

Research Design: - Cross sectional survey.

Research Variables

1) Dependant variables: Perception and behavior to ward covid-19 vaccine

2) Demographic variables: demographic variables of Nursing Student's such as Age, Gender, Course, Year, Marital status, vaccination history, side effects.

\section{Sampling method:-}

The E-survey was prepared online and hyperlink of the survey was distributed to students using mobile group messaging application. It was made sure in a class that most of the students are having smart mobile devices and sufficient Internet connectivity to fill up the form online. Students who were not using Internet were encouraged to take help from their friends having Internet enabled device. Prior to the distribution, students 
were made clear about the objectives of this study and inform consent form. It is to be noted that student participation was voluntarily and they could opted not to fill up the E-survey.

Instrument for Data Collection: For the data collection toll has been prepared in three categories. 1. Questionnaire related to Covid-19 vaccine $1^{\text {st }}$ dose, 2. Questionnaire related to Covid-19 vaccine $2^{\text {nd }}$ dose, and 3 point likert scale to assess the perception and behavior.

Study population:- Nursing College Students. And Faculties.

Study Sample: - Nursing students and faculties who received covid-19 vaccine

Study Setting: 04 nursing institutes of the kheda and Anand District Gujarat.

Sample Size: - 254 Nursing College Student and Faculties.

\section{SAMPLE CRITERIA}

Inclusion criteria:

1. Students and faculties of nursing colleges of both gender of age between 17- 60 years.

2. Those who have taken COVID - 19 vaccine.

\section{Exclusive criteria:}

1. Those who are not willing to participate in study.

2. Those who have not taken vaccine.

\section{Tool for Data Collection:}

Section-I: Consist of Demographic variables.

Section-II: Consist of Questionnaire related to Covid-19 vaccine $1^{\text {st }}$ dose.

Section-III: Consist of Questionnaire related to Covid-19 vaccine $2^{\text {nd }}$ dose.

Section-IV: Consist of 3 point likert scale to assess the perception and behavior.

\section{RESULTS}

Section I: Demographic variables of nursing students and faculties.

The [Table/Fig-1] portrays that majority participants (96\%) age below 25 years, majority $(86 \%)$ were female, $(70 \%)$ were undergraduate students.

[Table/Fig-1] depicts majority (87\%) were belong to Hindu religion, (89\%) were unmarried, $(95 \%)$ received vaccine free of cost, majority $(86 \%)$ taken vaccine empty stomach.

\begin{tabular}{|c|c|c|c|}
\hline Variables & Categories & (F) & $(\%)$ \\
\hline \multirow[t]{4}{*}{ Age } & $17-25$ & 245 & $96.45 \%$ \\
\hline & $26-35$ & 8 & $3.14 \%$ \\
\hline & $36-45$ & 0 & $0 \%$ \\
\hline & $46-60$ & 1 & $0.39 \%$ \\
\hline \multirow[t]{2}{*}{ Gender } & Male & 35 & $13.77 \%$ \\
\hline & Fe male & 219 & $86.22 \%$ \\
\hline \multirow{4}{*}{$\begin{array}{c}\text { Educational } \\
\text { status }\end{array}$} & Dip loma & 53 & $20.87 \%$ \\
\hline & Undergraduate & 178 & $70.08 \%$ \\
\hline & $\begin{array}{l}\text { Postgraduate } \\
\text { Degree }\end{array}$ & 23 & $9.05 \%$ \\
\hline & M.Phil./Ph.D. & 0 & $0 \%$ \\
\hline \multirow[t]{3}{*}{ Religion } & Hindu & 223 & $87.79 \%$ \\
\hline & Muslim & 5 & $1.97 \%$ \\
\hline & Christian & 26 & $10.24 \%$ \\
\hline \multirow[t]{3}{*}{ Marital Status } & Un married & 227 & $89.37 \%$ \\
\hline & Married & 24 & $9.45 \%$ \\
\hline & $\begin{array}{l}\text { Widow/Wido } \\
\text { wer }\end{array}$ & 3 & $1.18 \%$ \\
\hline \multirow[t]{2}{*}{ Residence } & Urban Area & 134 & $52.75 \%$ \\
\hline & Rural Area & 120 & $47.24 \%$ \\
\hline \multirow{5}{*}{$\begin{array}{l}\text { Family } \\
\text { Monthly } \\
\text { Income }\end{array}$} & Below 10,000 & 34 & $13.38 \%$ \\
\hline & $10,000-20,000$ & 57 & $22.44 \%$ \\
\hline & $20,001-30,000$ & 34 & $13.38 \%$ \\
\hline & Above 30,000 & 66 & $25.98 \%$ \\
\hline & I Don't know & 63 & $24.80 \%$ \\
\hline Sources of & Online media & 109 & $42.92 \%$ \\
\hline
\end{tabular}


medRxiv preprint doi: https://doi.org/10.1101/2021.07.18.21260710; this version posted July 29, 2021. The copyright holder for this preprint (which was not certified by peer review) is the author/funder, who has granted medRxiv a license to display the preprint in perpetuity.

It is made available under a CC-BY 4.0 International license.

\begin{tabular}{|c|c|c|c|}
\hline \multirow{2}{*}{$\begin{array}{c}\text { information } \\
\text { about COVID }\end{array}$} & Television & 44 & $17.32 \%$ \\
\cline { 2 - 4 } & News paper & 12 & $4.72 \%$ \\
\cline { 2 - 4 } & $\begin{array}{c}\text { Friends/Family } \\
\text { members }\end{array}$ & 14 & $5.51 \%$ \\
\cline { 2 - 4 } & Teachers & 51 & $20.07 \%$ \\
\cline { 2 - 4 } & Others & 24 & $9.44 \%$ \\
\hline \multirow{2}{*}{$\begin{array}{c}\text { Paid for } \\
\text { vaccine }\end{array}$} & Yes & 11 & $4.33 \%$ \\
\cline { 2 - 4 } $\begin{array}{c}\text { Negative } \\
\text { media } \\
\text { influence you }\end{array}$ & No & 243 & $95.67 \%$ \\
\cline { 2 - 4 } $\begin{array}{c}\text { Taken vaccine } \\
\text { empty } \\
\text { stomach? }\end{array}$ & Yes & 35 & $21.65 \%$ \\
\cline { 2 - 4 } & No & 219 & $86.22 \%$ \\
\hline
\end{tabular}

Table/Fig-1]: Frequency and percentage distribution according to demographic variables. $(\mathrm{N}=254)$

Key:- $(\mathrm{F})=$ Frequency, $(\%)=$ Percentage

\section{Section II: Distribution according to} information during $1^{\text {st }}$ dose of covid-19 vaccine.

The [Table/Fig-2] depicts majority (87\%) willingly taken vaccine, $(80 \%)$ were taken Covishield vaccine, (80\%) does not have any serious side effects after taken vaccine, (42\%) mild fever and last longer for 1-2 days. (43.70\%) had mild pain on the vaccine site, 203(79.92\%) participant do not have fear prior to take corona vaccine.

\begin{tabular}{|c|c|c|}
\hline Statement & Total & $\%$ \\
\hline $\begin{array}{l}\text { Status of COVID-19 vaccination? } \\
\text { Willingly taken } \\
\text { Not Willingly taken }\end{array}$ & $\begin{array}{c}221 \\
33\end{array}$ & $\begin{array}{l}87.00 \\
13.00\end{array}$ \\
\hline $\begin{array}{l}\text { Which COVID-19 Vaccine injected in } \\
\text { your body? } \\
\text { Covishield } \\
\text { Covaxin }\end{array}$ & $\begin{array}{c}205 \\
49\end{array}$ & $\begin{array}{l}80.7 \\
19.3\end{array}$ \\
\hline $\begin{array}{l}\text { Do you have any se vere side effects } \\
\text { after taking } 1^{\text {st }} \text { dose of COVID-19 } \\
\text { vaccine? } \\
\text { Yes } \\
\text { No }\end{array}$ & $\begin{array}{c}51 \\
203\end{array}$ & $\begin{array}{l}20.0 \\
80.0\end{array}$ \\
\hline $\begin{array}{l}\text { After taking } \mathbf{1}^{\text {st }} \text { dose of vaccine status of } \\
\text { fe ver? } \\
\text { Mild Fever } \\
\text { Moderate Fever } \\
\text { Severe Fever } \\
\text { None of above }\end{array}$ & $\begin{array}{l}109 \\
53 \\
18 \\
74\end{array}$ & $\begin{array}{c}42.9 \\
29.8 \\
7.0 \\
29.1\end{array}$ \\
\hline $\begin{array}{l}\text { Duration of Fe ver after taking } 1^{\text {st }} \text { dose } \\
\text { of COVID-19 vaccine? } \\
\text { 1-day }\end{array}$ & 97 & 38.1 \\
\hline
\end{tabular}

\begin{tabular}{|c|c|c|}
\hline $\begin{array}{l}\text { 2-days } \\
\text { 3-days or more } \\
\text { None of above }\end{array}$ & $\begin{array}{l}79 \\
08 \\
70 \\
\end{array}$ & $\begin{array}{c}31.1 \\
3.1 \\
27.7 \\
\end{array}$ \\
\hline $\begin{array}{l}\text { Did you required hos pitalization after } \\
\text { taking } 1^{\text {st }} \text { dose of COVID-19 vaccine? } \\
\text { Yes } \\
\text { No }\end{array}$ & $\begin{array}{c}10 \\
244 \\
\end{array}$ & $\begin{array}{r}4.0 \\
96.0 \\
\end{array}$ \\
\hline $\begin{array}{l}\text { Status of injection site pain after taking } \\
\mathbf{1}^{\text {st }} \text { dose of COVID-19 vaccine? } \\
\text { Mild Pain } \\
\text { Moderate Pain } \\
\text { Severe pain } \\
\text { None of above } \\
\end{array}$ & $\begin{array}{l}111 \\
65 \\
19 \\
59 \\
\end{array}$ & $\begin{array}{c}43.7 \\
25.5 \\
7.5 \\
23.2 \\
\end{array}$ \\
\hline $\begin{array}{l}\text { Duration of injection site pain after } \\
\text { taking } 1^{\text {st }} \text { dose of COVID-19 vaccine? } \\
\text { 1-day } \\
\text { 2-days } \\
\text { 3-days or more } \\
\text { None of above } \\
\end{array}$ & $\begin{array}{l}67 \\
68 \\
60 \\
59 \\
\end{array}$ & $\begin{array}{l}26.4 \\
26.8 \\
23.6 \\
23.2\end{array}$ \\
\hline $\begin{array}{l}\text { After taking } 1^{\text {st }} \text { dose of COVID-19 } \\
\text { vaccine which other side effects do you } \\
\text { have? } \\
\text { Nausea and vomiting } \\
\text { Lethargy } \\
\text { Headache and Body ache } \\
\text { None of above }\end{array}$ & $\begin{array}{c}05 \\
18 \\
131 \\
100\end{array}$ & $\begin{array}{l}2.0 \\
7.09 \\
51.1 \\
39.0\end{array}$ \\
\hline $\begin{array}{l}\text { Do you have fear prior to take COVID- } \\
19 \text { vaccine? } \\
\text { Yes } \\
\text { No }\end{array}$ & $\begin{array}{c}51 \\
203\end{array}$ & $\begin{array}{l}20.0 \\
80.0\end{array}$ \\
\hline
\end{tabular}

[Table/Fig-2] Frequency and percentage distribution according to information during $1^{\text {st }}$ dose of covid-19 vaccine $(\mathrm{N}=254)$.

Section III: Distribution according to information during $2^{\text {nd }}$ dose of covid-19 vaccine.

[Table/Fig-3] depicts majority (89\%) willingly taken vaccine, (90\%) does not have fear $(97 \%)$ does not have any serious side effects after taken vaccine, (37\%) mild fever and last longer for 1-2 days. (44.70\%) had mild pain on the vaccine site.

\begin{tabular}{|l|c|c|}
\hline \multicolumn{1}{|c|}{ Statement } & Total & \% \\
\hline Status of COVID-19 vaccination? & 226 & 89.00 \\
Willingly taken & 28 & 11.00 \\
Not Willingly taken & & \\
\hline Do you have fear to take 2 & \\
Yes & 27 & 10.0 \\
No & 227 & 90.0 \\
\hline $\begin{array}{l}\text { Do you have any se vere side effects } \\
\text { after taking 2nd dose of COVID-19 }\end{array}$ & & \\
vaccine? & 8 & 3.0 \\
Yes & 246 & 97.0 \\
\hline
\end{tabular}




\begin{tabular}{|c|c|c|}
\hline No & & \\
\hline $\begin{array}{l}\text { After taking } 2^{\text {nd }} \text { dose of vaccine status } \\
\text { of fe ver? }\end{array}$ & & \\
\hline Mild Fever & 95 & 37.4 \\
\hline Moderate Fever & 13 & 5.1 \\
\hline Severe Fever & 3 & 1.18 \\
\hline None of above & 143 & 55.12 \\
\hline $\begin{array}{l}\text { Duration of Fe ver after taking } 2^{\text {nd }} \text { dose } \\
\text { of COVID-19 vaccine? }\end{array}$ & & \\
\hline 1-day & 65 & 25.6 \\
\hline 2-days & 44 & 17.3 \\
\hline 3 -days or more & 4 & 2.0 \\
\hline None of above & 140 & 55.1 \\
\hline $\begin{array}{l}\text { Did you required hos pitalization after } \\
\text { taking } 2^{\text {nd }} \text { dose of COVID-19 vaccine? }\end{array}$ & & \\
\hline Yes & 8 & 3.1 \\
\hline No & 246 & 96.9 \\
\hline $\begin{array}{l}\text { Status of injection site pain after taking } \\
2^{\text {nd }} \text { dose of COVID-19 vaccine? }\end{array}$ & & \\
\hline Mild Pain & 114 & 44.9 \\
\hline Moderate Pain & 58 & 22.8 \\
\hline Severe pain & 03 & 1.18 \\
\hline None of above & 79 & 31.1 \\
\hline $\begin{array}{l}\text { Duration of injection site pain after } \\
\text { taking } 2^{\text {nd }} \text { dose of COVID-19 vaccine? }\end{array}$ & & \\
\hline 1-day & 86 & 33.8 \\
\hline 2-days & 70 & 27.5 \\
\hline 3-days or more & 15 & 6.0 \\
\hline None of above & 83 & 32.6 \\
\hline $\begin{array}{l}\text { After taking } 2^{\text {nd }} \text { dose of COVID-19 } \\
\text { vaccine which other side effects do you } \\
\text { have? }\end{array}$ & & \\
\hline Nausea and vomiting & 1 & 0.39 \\
\hline Lethargy & 7 & 2.75 \\
\hline Headache and Body ache & 88 & 34.6 \\
\hline None of above & 158 & 62.2 \\
\hline
\end{tabular}

[Table/Fig-3] Frequency and percentage distribution according to information during $2^{\text {nd }}$ dose of covid-19 vaccine $(\mathrm{N}=254)$.

Section IV: Distribution according to Perception and behavior toward covid-19 vaccine

[Table/Fig-4] depicts majority only $7(2.7 \%)$ had poor perception, 60(23.7\%) had moderate perception, 187(73.6) majority of participant had good perception.

\begin{tabular}{|c|c|c|}
\hline $\begin{array}{c}\text { Perception and } \\
\text { behavior level }\end{array}$ & Frequency & Percentage \\
\hline Poor Perception & 7 & 2.7 \\
\hline $\begin{array}{c}\text { Moderate } \\
\text { Perception }\end{array}$ & 60 & 23.7 \\
\hline Good Perception & 187 & 73.6 \\
\hline
\end{tabular}

\begin{tabular}{|c|c|c|}
\hline Total & 254 & $100 \%$ \\
\hline
\end{tabular}

[Table/Fig-4] Frequency and percentage distribution according Perception and behavior toward covid-19 vaccine.

[Table/Fig-5] Range, Mean, Standard deviation, Median score of perception and behavior among nursing students \& Faculties. $(\mathrm{n}=\mathbf{2 5 4})$

\begin{tabular}{|c|c|c|c|c|c|}
\hline \multirow{2}{*}{$\begin{array}{l}\text { Max. } \\
\text { Score }\end{array}$} & \multicolumn{5}{|c|}{ Perception and behavior Score } \\
\cline { 2 - 6 } & $\begin{array}{l}\text { Min- } \\
\text { Mx }\end{array}$ & Range & Mean & SD & Median \\
\hline 60 & $23-60$ & 37 & 48.68 & 7.32 & 50.50 \\
\hline
\end{tabular}

[Table/Fig-6] Level of Perception and behavior

toward covid-19 vaccine likert scale $(n=254)$

\begin{tabular}{|l|c|c|c|}
\hline \multirow{2}{*}{ Statement } & A & U & DA \\
\cline { 2 - 4 } & \multicolumn{2}{|c|}{ Percentage } \\
\hline $\begin{array}{l}\text { Will you reco mmend COVID 19 } \\
\text { vaccine to others? }\end{array}$ & 74.8 & 10.2 & 19.9 \\
\hline $\begin{array}{l}\text { Getting myself vaccinated for } \\
\text { COVID 19, would be good way to } \\
\text { protect myself against infection? }\end{array}$ & 81.4 & 10.2 & 8.2 \\
\hline $\begin{array}{l}\text { Does COVID 19 symptoms after } \\
\text { taken vaccine may differ from one } \\
\text { person to another? }\end{array}$ & 41.7 & 26.3 & 31.8 \\
\hline $\begin{array}{l}\text { Do you think COVID 19 vaccination } \\
\text { is an effective way to prevent and } \\
\text { control COVID 19? }\end{array}$ & 74.8 & 12.5 & 12.5 \\
\hline $\begin{array}{l}\text { Is Corona infection providing better } \\
\text { immunity than COVID 19 vaccine? }\end{array}$ & 44.8 & 24.0 & 31.1 \\
\hline Is COVID 19 vaccine safe for all? & 56.2 & 19.2 & 24.4 \\
\hline $\begin{array}{l}\text { Do you think all has to take COVID } \\
\text { 19 vaccine? }\end{array}$ & 68.1 & 16.9 & 14.9 \\
\hline COVID 19 vaccine may be fatal? & 9.05 & 44.8 & 46.0 \\
\hline $\begin{array}{l}\text { COVID 19 vaccine can lead to } \\
\text { serious health issues? }\end{array}$ & 7.8 & 24.8 & 67.3 \\
\hline $\begin{array}{l}\text { COVID 19 vaccine is sufficient for } \\
\text { preventing Corona virus to all? }\end{array}$ & 45.2 & 22.8 & 31.8 \\
\hline $\begin{array}{l}\text { I believe a vaccine can help control } \\
\text { the spread of COVID 19? }\end{array}$ & 23.6 & 60.6 & 15.7 \\
\hline $\begin{array}{l}\text { Is COVID 19 vaccine beco me } \\
\text { available, it should be mandatory for } \\
\text { all? }\end{array}$ & 55.9 & 23.6 & 20.4 \\
\hline $\begin{array}{l}\text { Is COVID 19 vaccine will be given to } \\
\text { everyone simu ltaneously? }\end{array}$ & 56.2 & 28.3 & 15.3 \\
\hline Is it mandatory to take the vaccine? & 50 & 27.9 & 22.0 \\
\hline $\begin{array}{l}\text { Is it neces sary for a COVID } \\
\text { recovered person to take the vaccine? }\end{array}$ & 57.0 & 27.5 & 15.3 \\
\hline
\end{tabular}




\begin{tabular}{|l|c|c|c|}
\hline $\begin{array}{l}\text { The vaccine introduced in India be as } \\
\text { effective as the ones introduced in } \\
\text { other countries? }\end{array}$ & 53.9 & 35.8 & 10.2 \\
\hline $\begin{array}{l}\text { Does one need to follow preventive } \\
\text { measures such as wearing mask, hand } \\
\text { sanitization, social distancing after } \\
\text { receiving the COVID 19 vaccine? }\end{array}$ & 72.4 & 15.7 & 11.8 \\
\hline $\begin{array}{l}\text { Are there any common side effects of } \\
\text { this vaccine? }\end{array}$ & 53.1 & 14.5 & 32.2 \\
\hline $\begin{array}{l}\text { Does negative media information's } \\
\text { work as a barrier in COVID 19 } \\
\text { vaccination? }\end{array}$ & 47.6 & 25.1 & 27.1 \\
\hline $\begin{array}{l}\text { Will you follow all the COVID 19 } \\
\text { protocols once you get vaccinated? }\end{array}$ & 73.6 & 17.3 & 9.0 \\
\hline
\end{tabular}

Key:- A:Agree, U:Undecided, DA: Disagree

Section V: Distribution according to Association between perception and selected demographic variables.

The [Table/Fig-7] depicts outcome of chi-square test results, In reference to the association of perception and behaviour with selected demographic variables, there was significant association of perception with sources of corona vaccine information and rest of variable found not significant, at 0.05 level of significant.

\begin{tabular}{|c|c|c|c|c|}
\hline Variables & Categories & (f) & $\begin{array}{c}\text { Chi- } \\
\text { Square }\end{array}$ & $\begin{array}{l}\text { Sig. P- } \\
\text { val ve }\end{array}$ \\
\hline \multirow{4}{*}{ Age } & $17-25$ & 245 & \multirow{2}{*}{4.063} & \multirow{4}{*}{$\begin{array}{l}0.907 \\
\mathrm{NS}\end{array}$} \\
\hline & $26-35$ & 8 & & \\
\hline & $36-45$ & 0 & \multirow[b]{2}{*}{$\mathrm{df}=9$} & \\
\hline & $46-60$ & 1 & & \\
\hline \multirow{2}{*}{ Gender } & Male & 35 & 0.916 & 1.00 \\
\hline & Female & 219 & $\mathrm{Df}=9$ & NS \\
\hline \multirow{4}{*}{$\begin{array}{c}\text { Education } \\
\text { al status }\end{array}$} & Dip loma & 53 & \multirow{4}{*}{$\begin{array}{l}5.89 \\
\mathrm{df}=9\end{array}$} & \multirow{4}{*}{$\begin{array}{l}0.750 \\
\text { NS }\end{array}$} \\
\hline & Undergraduate & 178 & & \\
\hline & $\begin{array}{c}\text { Postgraduate } \\
\text { Degree }\end{array}$ & 23 & & \\
\hline & M.Phil./Ph.D. & 0 & & \\
\hline \multirow{3}{*}{ Religion } & Hindu & 223 & \multirow{3}{*}{$\begin{array}{l}5.278 \\
\mathrm{Df}=9\end{array}$} & \multirow{3}{*}{$\begin{array}{l}0.908 \\
\text { NS }\end{array}$} \\
\hline & Muslim & 5 & & \\
\hline & Christian & 26 & & \\
\hline \multirow{3}{*}{$\begin{array}{l}\text { Marital } \\
\text { Status }\end{array}$} & Un married & 227 & \multirow{3}{*}{$\begin{array}{l}1.891 \\
\mathrm{df}=9\end{array}$} & \multirow{3}{*}{$\begin{array}{l}0.993 \\
\text { NS }\end{array}$} \\
\hline & Married & 24 & & \\
\hline & Widow & 3 & & \\
\hline \multirow[b]{2}{*}{ Residence } & Urban Area & 134 & \multirow[t]{2}{*}{4.046} & \multirow{2}{*}{$\begin{array}{l}0.67 \\
\text { NS }\end{array}$} \\
\hline & Rural Area & 120 & & \\
\hline \multirow{5}{*}{$\begin{array}{l}\text { Family } \\
\text { Monthly } \\
\text { Income }\end{array}$} & Be low 10,000 & 34 & \multirow{5}{*}{$\begin{array}{l}19.86 \\
\mathrm{df}=15\end{array}$} & \multirow{5}{*}{0.177} \\
\hline & $10,000-20,000$ & 57 & & \\
\hline & $20,001-30,000$ & 34 & & \\
\hline & Above 30,000 & 66 & & \\
\hline & I Don't know & 63 & & \\
\hline
\end{tabular}

\begin{tabular}{|c|c|c|c|c|}
\hline \multirow{3}{*}{$\begin{array}{c}\text { Sources of } \\
\text { informatio }\end{array}$} & Online media & 109 & & \\
\cline { 2 - 3 } n about & Television & 44 & \multirow{4}{*}{32.87} & $\mathbf{0 . 1 8}$ \\
\cline { 2 - 3 } $\begin{array}{c}\text { COVID 19 } \\
\text { vaccine }\end{array}$ & $\begin{array}{c}\text { News paper } \\
\text { Fiends/Family } \\
\text { me mbers }\end{array}$ & 12 & $\begin{array}{c}\text { Signific } \\
\text { ant }\end{array}$ \\
\cline { 2 - 3 } & Teachers & 51 & & \\
\cline { 2 - 3 } & Others & 24 & & \\
\hline
\end{tabular}

[Table/Fig-7] outcome of chi-square test results to find significant association between selected demographic variables of nursing students and faculties $(n=254)$

Conclusion: On the basis of findings of this study the following conclusion were drawn:

The purpose of the present study is to assess the attitude regarding online lecture after the impact of COVID-19 at selected nursing college Nadiad. The study consisted of 136 samples that were selected on the basis of simple randomization techniques. Based on the objective,

the data analysis was done by calculating the mean, percentage , standard deviation. Result revealed that an attitude of nursing students towards Regarding the choice of gadgets to attends online class of students out of 136 samples, $132(97.05 \%)$ was mobile, $2(1.47 \%)$ was laptop2(1.47\%). Regarding the network quality of students out of 136 samples, 33(24.26\%) was poor, $79(58.08 \%)$ was average, $24(17.64 \%)$ good. Revealed that the distribution of sample according to Attitude regarding Online Classes were 7 (5.14\%) had Inadequate Attitude 86(63.25\%) had Moderate Attitude, 43(31.61\%) Had adequate attitude.

Conflict of Interest: Nil (there is not any conflict of interest between the all authors)

Source of Funding: Self (Contributed by all authors)

Ethical Clearance: The study was approved by the institutional ethical committee of Dinsha Patel 
college of nursing, research committee, There are total 15 members in the committee from various field. The ethical approval reference number is DPCN/2 ${ }^{\text {nd }}$ IEC/2020-21/13 and a formal written permission was gathered from the authority of or Principal of Institute prior to data collection

Statement of Informed consent: Yes informed consent form was acquired from the participants prior to data collection.

\section{Acknowledgement}

Special thanks to all the participants of the study and principals of the selected colleges for provide us permission for data collection.

\section{REFERENCES}

1. Corona virus pandemic, WHO, [internet] available from Coronavirus disease (COVID-19) (who.int)

2. Introduction to COVID-19, Physiopedia, [Internet] available from Coronavirus Disease (COVID-19) Physiopedia (physio-pedia.com)

3. About corona virus cases in 2021 [online] available from url: https://www.aljazeera.com/news/2021/1/26/globalnumber-covid-cases-surpasses-100-million

4. Andrews MA, Areekal B, Rajesh KR, et al. First confirmed case of COVID-19 infection in India: A case report. Indian J Med Res. 2020;151(5):490492. doi:10.4103/ijmr.IJMR_2131_20, available from url: https://www.ncbi.nlm.nih.gov/pmc/articles/PMC75 $\underline{30459 /}$

5. Funk CD, Laferrière C and Ardakani A (2020) A Snapshot of the Global Race for Vaccines Targeting SARS-CoV-2 and the COVID-19 Pandemic. Front. Pharmacol. 11:937. doi: 10.3389/fphar.2020.00937, [internet] Available from Url: https://www.frontiersin.org/articles/10.3389/fphar. $\underline{2020.00937 / f u l l}$

6. COvid-19 vaccination in India, [internet] available from Url: https://www.mohfw.gov.in/pdf/COVID19Vac cinationGuide forHealthcareandFrontline Work $\underline{\text { ers.pdf }}$

7. Kailash Nagar, Knowledge, Attitude and Practice on Personal Hygiene among School Children in Rural Primary School of Kheda District, Gujarat. Indian Journal of Forensic Medicine \& Toxicology [Internet]. 2021May17,15(3):290-5. Available from:http://medicopublication.com/index.php/ijfmt /article/view/15321

8. Kailash Nagar, Assess The Attitude Regarding Online Lecture Among Nursing College Students After Impact of Covid-19, Medrxiv publication, [Internet] Available from: doi: https://doi.org/10.1101/2021.07.01.21259132

9.

10. Ahmad Mehmood, Beg Bilal Mahmood, et.all Epidemiological and Clinical Characteristics of COVID-19: A Retrospective Multi-Center Study in Pakistan, Frontiers in Public Health, volume:9 2021 page-342, Url Frontiers 1 Epidemiological and Clinical Characteristics of COVID-19: A Retrospective Multi-Center Study in Pakistan Public Health (frontiersin.org) DOI: https://doi.org/10.3389/fpubh.2021.644199

11. Covid-19 vaccines [Internet] available from url; https://www.mohfw.gov.in/pdf/COVID19Vaccine OG111Chapter16.pdf

12. Patidar K, Sharma M, Gautam A, Sharma DK, Jain J. COVID-19 Knowledge and Perception among Budding Nurses: A QuestionnaireBased Survey. Int J Nur Res. 2020; 6(2): 1-7 [internet] available from url: International Journal of Nursing Research (IJNR) (innovationa lpublishers.com)

DOI: $10.31690 / \mathrm{ijnr} / 61$

13. WHO Draft Landscape and Tracker of COVID-19 Candidate Vaccines. [(accessed on 27 February 2021)]; Available online: COVID-19 vaccine tracker and landscape (who.int)

14. Md. Saiful Islam, Abu Bakkar Siddique, Knowledge, attitudes and perceptions towards COVID19 vaccinations: a cross-sectional community survey in Bangladesh, medrxiv, [Internet] Available from url: Knowledge, attitudes and perceptions towards COVID-19 vaccinations: a cross-sectional community survey in Bangladesh $\mid$ medRxiv doi: https://doi.org/10.1101/2021.02.16.21251802 
medRxiv preprint doi: https://doi.org/10.1101/2021.07.18.21260710; this version posted July 29, 2021. The copyright holder for this preprint (which was not certified by peer review) is the author/funder, who has granted medRxiv a license to display the preprint in perpetuity.

It is made available under a CC-BY 4.0 International license .

15. Khan Sharun1, C.K. Faslu Rahman et. All, covid19 vaccine acceptance: beliefs and barriers associated with vaccination among the general population in India, Journal of Experimental Biology and Agricultural Sciences, December 2020; Volume - 8(Spl-1- SARS-CoV-2) page S210 - S218, Available from url: 439_pdf.pdf (jebas.org) DOI:10.18006/2020.8(Spl-1-SARSCoV-2).S210.S218

16. Modern, Covovax, Biological E: What we know about India's new Covid vaccines, Available from Url: Moderna, Covovax, Biological E: What we know about India's new Covid vaccines - BBC News

17. India's First Indigenous COVID-19 Vaccine, Bharat biotech available from url: COVAXIN -

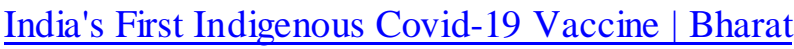
Biotech 